Anaesthesist 2016 65:653-654

DOI 10.1007/s00101-016-0204-1

Online publiziert: 22. Juli 2016

๑) Springer-Verlag Berlin Heidelberg 2016

CrossMark

Deutschland legt zu: Nach Angaben der Deutschen Adipositas-Gesellschaft [2] hat die Prävalenz von Übergewicht und Adipositas in den vergangenen Jahren in Deutschland (und weltweit) kontinuierlich zugenommen. Knapp $60 \%$ der deutschen Bevölkerung sind übergewichtig (Body-Mass-Index [BMI] $\geq 25 \mathrm{~kg} / \mathrm{m}^{2}$ : Anteil = $37 \%$ ) oder adipös $\left(\mathrm{BMI} \geq 30 \mathrm{~kg} / \mathrm{m}^{2}\right.$ : Anteil = $\left.21 \%\right)$.

Entsprechend steigt die Anzahl kritisch kranker Patienten mit Adipositas auf Intensivstationen. Sie werden dort zumeist mit besonderer Aufmerksamkeit behandelt [7] und stellen eine spezielle Herausforderung sowohl bezüglich des praktischen Managements (geeignete Betten-, Lagerungs- oder Mobilisierungssysteme) als auch bezüglich der diagnostischen (Computertomographie, Ultraschall) und medizinischen Herangehensweise (Beatmung, Ernährung, Medikamentendosierung) dar.

\section{Das „obesity paradoxon"}

Obwohl schwer adipöse Intensivpatienten generell von mehr und schwerwiegenderen Komplikationen bedroht sind (Infektionen, Thrombosen, hämodynamische Instabilität, Nierenfunktionsstörung), wurde durch große Kohortenstudien aufgezeigt, dass adipöse Patienten eine niedrigere vergleichbare Letalität aufweisen als normal- oder untergewichtige kritisch Kranke. Bei ca. 10.000 Patienten operativer Intensivstationen [3] und bei 1909 Patienten nach koronarer Bypassoperation [4] wurde ein Überlebensvorteil für übergewichtige oder adipöse Patienten gefunden. Die Bedeutung dieser auf den ersten Blick überraschenden Befunde (,obesity paradoxon") ist bisher nicht klar. Es wird zum einen vermutet, dass adipöse Patienten wegen ihrer Körperkonfiguration eine

\title{
T. Bein
}

Klinik für Anästhesiologie, Universitätsklinikum Regensburg, Regensburg, Deutschland

\section{Die dunkle Seite der Adipositas}

besonders achtsame Zuwendung von Ärzten und Pflegenden erfahren und einen Therapievorteil besitzen [1]. Zum anderen wird postuliert, dass Adipozyten eine moderate antiinflammatorische Funktion ausüben und bei kritischer Erkrankung „protektiv“ wirken [5]. Beide Hypothesen sind bisher nicht durch ausreichende klinische Daten gestützt.

\section{Fett ist nicht gleich Fett}

Eine dritte Hypothese - und diese wird durch die Übersicht von Weig et al. in dieser Ausgabe von Der Anaesthesist [9] unterstrichen - sieht den bisher für alle wissenschaftlichen Arbeiten herangezogenen Body-Mass-Index als unspezifisches und nicht ausreichend valides Instrument zur Beschreibung der Adipositas an. Weig et al. hatten in einer retrospektiven Kohorte von ARDS-Patienten die Beobachtung gemacht, dass einige adipöse Patienten, die wegen eines schweren ARDS mit prolongierter Bauchlagerung behandelt wurden, eine assoziierte Verschlechterung der Funktion intestinaler Organe aufwiesen [8]. Die nähere Analyse zeigte, dass diese gravierenden Funktionseinschränkungen (Nierenversagen in $83 \%$, hypoxämische Hepatitis in $22 \%$ ) überwiegend Patienten mit abdomineller Adipositas zuzuordnen waren, während adipöse Patienten mit vergleichbar erhöhtem BMI, die aber ein subkutanes Fettverteilungsmuster aufwiesen, eine signifikant geringere Inzidenz der bauchlagerungsassoziierten Komplikationen (Nierenversagen $35 \%$, Hepatitis $2 \%$ ) hatten. Diese pathophysiologisch und klinisch bedeutsamen Befunde lehren: BMI ist nicht gleich BMI und Adipositas ist nicht gleich Adipositas. Offensichtlich ist das Verteilungsmuster der Adipositas - subkutan vs. abdominell - von großer Bedeu- tung für die Risikoeinschätzung kritisch kranker Patienten. In ähnlicher Weise wird schon seit Langem auf das hohe Letalitätsrisiko bei Vorliegen eines metabolischen Syndroms hingewiesen [6]: Abdominelle Adipositas, Hypertonus, Diabetes mellitus und erhöhtes Cholesterin gelten als „tödliches Quartett“.

Die vorliegende Übersicht von Weig et al. beschreibt die für Anästhesie und Intensivmedizin bedeutsamen Methoden, dieklinischen Aspekte und mögliche Fallstricke der Einschätzung der Adipositas. Sie legt besonderen Wert auf die Notwendigkeit der differenzierten Einschätzung des Adipositastyps und der daraus resultierenden Konsequenzen für die Therapiestrategie. Die hierfür notwendigen diagnostischen Instrumente (Schublehre zur Erfassung des sagittalen abdominellen Diameters, Computertomogramm) dürften den meisten Intensivstationen ohne großen zusätzlichen Aufwand zur Verfügung stehen. In diesem Zusammenhang zeigen die Autoren auch eine attraktive Methode zur generellen Einschätzung der Muskelmasse am Beispiel des M. psoas auf. Auch dieser Aspekt ist sowohl im Zusammenhang mit Adipositas als auch für sich gesehen („ICU-acquired weakness") ein wichtiger Parameter zur Erfassung des funktionellen Outcomes nach Intensivbehandlung.

Die Übersicht von Weig et al. ist somit ein Baustein zur „individualisierten“ medizinischen Behandlung, die auch in der Intensivtherapie zunehmend gefordert wird: Der Weg führt weg von globalen Diagnosen (Adipositas, ARDS, Sepsis, Organversagen, Trauma, akutes Abdomen) zu einer mittels sorgfältiger Anamnese, klinischer und bildgebender Untersuchung und durch gezielt und kenntnisreich erhobene pathophysiologische Daten abgestützten Wahrnehmung der 
- oft komplexen - individuellen Erkrankung des Patienten mit daraus resultierender „maßgeschneiderter“ Therapie.

Der Anfang zur differenzierten Einschätzung der Adipositas ist getan - der wissenschaftliche Weg zur Untersuchung weiterer Aspekte der dunklen Seite der Adipositas liegt noch vor uns!

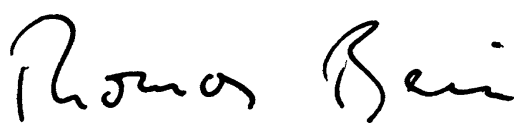

\section{Korrespondenzadresse}

\section{Prof. Dr. T. Bein, M.A.}

Klinik für Anästhesiologie, Universitätsklinikum Regensburg

93042 Regensburg, Deutschland

thomas.bein@ukr.de

Interessenkonflikt. T. Bein gibt an, dass kein Interessenkonflikt besteht.

\section{Literatur}

1. Carrara FS, Zanei SS, Cremasco MF, Whitaker IY (2016) Outcomes and nursing workload related to obese patients in the intensive care unit. Intensive Crit Care Nurs 35:45-51

2. Deutsche Adipositas Gesellschaft www. adipositas-gesellschaft.de/index.php?id=41. Zugegriffen:20. Juni 2016

3. Hutagalung R, Marques J, Kobylka K, Zeidan M, Kabisch B, Brunkhorst F, Reinhart K, Sakr Y (2011) The obesity paradox in surgical intensive care unit patients. Intensive Care Med 37:1793-1799

4. Le-Bert G, Santana O, Pineda AM, Zamora C, Lamas GA, Lamelas J (2011) The obesity paradox in elderly obese patients undergoing coronary artery bypass surgery. Interact Cardiovasc Thorac Surg 13:124-127

5. Morin SO, Poggi M, Alessi MC, Landrier JF, Nunes JA (2016) Modulation of T cell activation in obesity. Antioxid Redox Signal. PMID 27225042. (Epub ahead of print)

6. O'Neill S, O'Driscoll L (2015) Metabolic syndrome: a closer look at the growing epidemic and its associated pathologies. Obes Rev 16:1-12

7. Shashaty MG, Stapleton RD (2014) Physiological and management implications of obesity in critical illness. Ann Am Thorac Soc 11:1286-1297

8. Weig T, Janitza S, Zoller M, Dolch ME, Miller J, Frey L, Kneidinger N, Johnson T, Schubert MI, Irlbeck M (2014) Influence of abdominal obesity on multiorgan dysfunction and mortality in acute respiratory distress syndrome patients treated with prone positioning. J Crit Care 29:557-561

9. Weig T, Irlbeck T, Frey L, Paprottka P, Irlbeck M (2016) Jenseits des BMI-Alternative Methoden zur Erfassung von Körperfett und Muskelmasse in der Intensivmedizin und deren klinischer Stellenwert. Anaesthesist.doi:10.1007/s00101-016-0205-0

\section{Raschka, L. Nitsche}

\section{Praktische Sportmedizin}

Stuttgart: Georg Thieme Verlag 2016, 328 S., 120 Abb., (ISBN 9783131756114 ), 59.99 EUR

Die Sportmedizin wird 2016 durch eine weitere Monographie ergänzt. Zeitgemäß wird auch ein Online-Zugang bzw. ein eBook bereitgestellt. Das Format der gebundenen Ausgabe ist mit $18 \mathrm{~cm} \times 24,5 \mathrm{~cm}$ sehr handlich, dadurch eignet sich das Buch auch als Reiselektüre hervorragend.

Die Anordnung der Kapitel ist logisch. Beginnend mit der sportmedizinischen Untersuchung, der Leistungs- und Funktionsdiagnostik, Sport bei spezifischen Erkrankungen und weiteren wichtigen Themen wie Ernährung und Doping, werden auch die Begleitgebiete Leistungsvergütung sportmedizinischer Untersuchungen, Sportlerbetreuung und rechtliche Aspekte abgehandelt.

Die Autoren bemühen sich das jeweilige Thema fokussiert für den „Kliniker“ vorzustellen. Die Formulierungen sind für den sportmedizinischen „Generalisten" verständlich und prägnant.

Die einzelnen Kapitel werden durch farbliche und gut verständliche Abbildungen sinnvoll ergänzt. Durch farblich markierte Textfelder werden wichtige Gesichtspunkte in die Kategorien „Merke“, „Praxistipp“ und "Cave!" didaktisch einprägsam hervorgehoben. In ausgewählten Kapiteln werden auch Fallbeispiele herangezogen, die die Thematik gemäß dem Buchtitel verdeutlichen sollen. Leider wird dieses didaktische Mittel vor allem für den Themenschwerpunkt "Leistungs- und Funktionsdiagnostik" nicht konsequent fortgeführt. Dasselbe trifft auch für eine Zusammenfassung am Ende eines Kapitels zu. Eine strikte Umsetzung nach jedem Kapitel hätte sich positiv auf die Wissensvermittlung ausgewirkt. Die Sportmedizin ist eine medizinische Disziplin, die sich aus zahlreichen medizinischen Fachgebieten (z.B. Orthopädie und Kardiologie) und mittelbar verbundenen Wissenschaften (Ernährungswissenschaften und Psychologie) zusammensetzt. Dies führt unweigerlich dazu, dass eine allumfassende Abhandlung der Sportmedizin nur schwer zu realisieren ist.
Dies trifft auch für diese Monographie zu. Allerdings entspricht dies auch nicht der Zielsetzung. Die „Praktische Sportmedizin“ versteht sich als Abhandlung für den Facharzt mit speziellem Interesse für die Sportmedizin.

Das Buch vermittelt einen schnellen und verständlichen Überblick über die Schnittstelle Sportmedizin im Gesamtkonzept der medizinischen Fachgebiete.

Relevant und interessant sind für den Leser eines Fachgebiets vor allem die sog. „fachfremden" Kapitel, da wichtige Aspekte und Hinweise präsentiert werden. Hierdurch erschließt sich dem jeweiligen Leser, aufgrund seiner individuellen Vorkenntnisse, sportmedizinisch relevantes, neues Wissen auf einprägsame Weise.

Die „Praktische Sportmedizin“ stellt ein „praxisorientiertes“ Kompendium für den "Generalisten" mit Interesse für die Sportmedizin dar und eignet sich auch begleitend zur Vorbereitung auf den Erwerb der Zusatzbezeichnung.

C. Spies (Bad Rappenau) 